\title{
Perceptions of Perinatal Loss: Miscarriage versus Stillbirth
}

\author{
Jane Plagge ${ }^{1}$, Pacific University, Oregon \\ Jennifer R. Antick, Pacific University, Oregon \\ KEY WORDS: PERINATAL LOSS, PREGNANCY LOSS, \\ MISCARRIAGE, STILLBIRTH, SOCIAL SUPPORT
}

\begin{abstract}
Lack of social support lengthens and intensifies the grieving process for women experiencing perinatal loss and is a predictor of depression as long as a year after loss. Support appears to be offered differentially based on gestational age. This study represents an attempt to assess biases that might hinder support. Female university students' perceptions of perinatal loss were ascertained using a hypothetical vignette about a close, personal friend who experiences either stillbirth (group 1) or miscarriage (group 2). They were all asked about expectations and beliefs as well as how they might personally react. The participants rating the stillbirth vignette reflected expectations of more grief and more personal discomfort than those rating the miscarriage vignette. The data from this study support previous findings regarding social support and perinatal loss. Limits, implications and future directions are discussed.
\end{abstract}

Perinatal loss, which includes miscarriage, stillbirth and neonatal death is fairly common (Côté-Arsnault, Bidlack \& Humm, 2001). In 2002, there were 6.4 per 1000 fetal deaths (20 weeks gestation or greater) and 4.7 neonatal deaths per 1000 live births in the United States (Kochanek et al., 2004). Researchers have reported that $15 \%$ to $20 \%$ of known pregnancies result in miscarriage (Van \& Meleis, 2002) and that early cases of miscarriage are often statistically underreported (Wallerstedt, Lilley, Baldwin, 2003). Fifteen percent to $25 \%$ of women who experience perinatal loss bear enduring adjustment problems and several seek professional help (Bennett et al., 2005). Clinicians and researchers suggest that perinatal loss places parents at risk for mental health complications, such as traumatic grief, depression, anxiety, adjustment

\footnotetext{
${ }^{1}$ Portions of this paper were presented at the Annual Meeting of the Western Psychological Association, May, 2007, Vancouver, BC. Inquiries about the manuscript should be forwarded to Dr. Jennifer R. Antick, 511 SW 10th, Suite 400, Portland, Oregon 97205-2732. (e-mail: jantick@pacificu.edu)
} 
disorder and posttraumatic stress disorder (PTSD), (Bennett et al., 2005). Turton, Hughes, Evans and Fainman (2001) estimated the lifetime risk for PTSD following perinatal loss as $29 \%$ and the risk for PTSD exhibited in a subsequent pregnancy as $20 \%$. Moreover, researchers (Hughes et al., 2002) reported that approximately $20 \%$ of women who experience stillbirth have prolonged depression and an additional 20\% develop PTSD.

More intense and prolonged grief reactions have been detected in women who received less support from their social network (Hughes \& Riches, 2003; Janssen et al., 1997). In fact, Forrest, Standish and Baum (1982) showed that social isolation and poor relations are correlated with high grief responses following perinatal loss. Additionally, Cuisinier, Kuijpers, Hoogduin, de Graauw and Janssen (1993) found that social support was significantly negatively correlated with grief intensity, such that as social support increased grief intensity decreased.

Researchers (Madden, 1994; Swanson, 2000) have also found correlations between social support and the length of the grieving process. Madden (1994) found that emotional intensity was negatively correlated with overall support from others and that intense emotions lasted as long as four months following miscarriage. Swanson (2000) further found low social support to be one of the strongest predictors of a decline in a woman's emotional strength and an increase in her tendency to cope passively and feel depressed as long as a year after miscarriage. Others have additionally identified social support as effective coping strategies following perinatal loss (Van \& Meleis, 2002).

Unfortunately, researchers (Bennett et al., 2005) have reported that society often views perinatal loss, contrary to other child losses, as insignificant which is likely to leave mothers feeling alone and invalidated in their grief. Rajan (1994) pointed out that women may discover that pregnancy losses are not always viewed by others as significant emotional experiences. Kavanagh (2002) further reported that people may think "because the baby never lived that it had no real existence or designated place in society" (p. 18). Mothers of stillborn infants, for example, have found their need to grieve goes unrecognized in that stillbirth has been described as a nonevent (Kavanagh, 2002; Rajan, 1994). Kavanagh reported that mothers have a great need to grieve for their lost child who lived inside them and Rajan stated that others' beliefs of stillbirth as a nonevent may subject the parents to feelings of guilt and shame.

Bennett and colleagues (2005) reported that others may feel uncomfortable discussing the loss with the family and Kavanagh (2002) further reported that society often compounds the grief and isolation of bereaved mothers by people's reluctance to speak of the loss of the infant. Bennett and colleagues (2005) suggested that most people have no 
prior knowledge or experiential history of perinatal loss and, therefore, it can be difficult for them to empathize with the parents. The authors also noted that family and friends who were supportive during the pregnancy, may withdraw their support from bereaved parents because they may not know how to offer condolences or manage the loss. Rajan (1994) further reported that overall people are awkward with and lack understanding for those who have experienced perinatal loss. The researcher reported that people do not know how to respond and are unable to easily relate to parents who have experienced a loss and therefore, a common reaction is to say nothing. One of the most frequent complaints expressed by the women in Rajan's study was that others, whom the women had considered friends, ignored them entirely.

Van and Meleis (2002) found that women who engaged in social support as a coping strategy following perinatal loss reported appreciation for the support as well as the significance it played in their recovery. The women noted the importance of the confirmation they received from people with whom they were able to talk about their loss. Family and friends were most often identified as those who women spoke with following their loss. The participants described supportive family and friends as reassuring and comforting and emphasized the importance of having someone to listen to them without judgment or criticism. Some participants reported that when they talked about their perinatal loss they felt relieved. The authors reported that talking to others seemed to help the women gain a better understanding of their experience and encouraged a sense of healing. In fact, when friends and family were supportive and available to the women in Van and Meleis' (2002) study, their presence was so significant that the women felt no need to seek additional aid through counseling or support groups. Women have reported finding their partners, mothers, extended families, friends and midwives as most helpful at the time of their pregnancy loss (DiMarco et al., 2001; Rajan, 1994). Additionally, many women have reported exchanges with other women who had suffered a similar loss as helpful and many of the parents within the studies further reported that they found those who were present and listened to be most helpful (DiMarco et al., 2001; Rajan, 1994).

\section{Purpose of the Study}

Van and Meleis (2002) reported that society provides more support for women who experience a late pregnancy loss, such as stillbirth, than for women who experience early pregnancy loss, such as miscarriage. Researchers (Cuisinier et al., 1993; Van \& Meleis, 2002) have suggested that society tends to view stillbirth as a more significant loss than miscarriage. However, it remains unclear whether women's social support networks actually perceive miscarriage as less significant than 
stillbirth and, therefore, provide varying degrees of support based on gestational age at the time of loss.

This study focused on perceptions of women's social support networks in regard to whether perinatal loss is viewed as more severe with increasing gestational age. The differences in expected grief of women who have experienced a miscarriage compared with women who have experienced a stillbirth were examined. It was hypothesized that (1) participant ratings of the stillbirth vignette would reflect higher scores on a measure of perceived grief than participant ratings of the miscarriage vignette and (2) participant ratings of the stillbirth vignette would reflect higher scores on an exploratory scale of expected discomfort visiting the woman than participant ratings of the miscarriage vignette.

\section{METHOD}

\section{Participants}

A total of 169 questionnaires were collected, however data from one participant was not included in the analysis of expected discomfort due to incomplete data. Eighty-four women reviewed the miscarriage vignette $(49.7 \%)$, whereas 85 women reviewed the stillbirth vignette (50.3\%). Participants' mean age was 27.21 years and their median age was 25 with a range of 20 to 55 . The majority of participants were graduate students $(73.2 \%)$, in which $46.6 \%$ were studying clinical psychology, $28 \%$ were studying counseling psychology and $3.1 \%$ were studying physical therapy. The remaining $26.8 \%$ of participants were undergraduate students, in which $18.8 \%$ indicated a liberal arts major and $8 \%$ indicated a science major. In regard to race and ethnicity, $84.6 \%$ of participants were Caucasian, $4.1 \%$ were Asian-American, $2.4 \%$ were Hispanic or Latina, $1.8 \%$ were African-American and $7.2 \%$ indicated other.

One-hundred and fifty-four participants (91.1\%) reported no personal history of perinatal loss, whereas, nine participants (5.3\%) reported history of miscarriage, four participants $(2.4 \%)$ reported history of other loss (excluding miscarriage, stillbirth and neonatal death) and two participants $(1.2 \%)$ reported experiencing more than one type of perinatal loss (miscarriage, stillbirth, neonatal death and/or other). Onehundred and thirty-eight participants $(81.7 \%)$ reported knowing someone with a history of perinatal loss. Of these, 80 participants $(47.3 \%)$ reported knowing someone with a history of miscarriage, 10 (5.9\%) reported knowing someone with a history of stillbirth, three $(1.8 \%)$ reported knowing someone with a history of neonatal death, eight $(4.7 \%)$ reported knowing someone with a history of other loss (excluding miscarriage, stillbirth and neonatal death) and 37 (21.9\%) reported 
Plagge \& Antick: PERCEPTIONS OF PERINATAL LOSS 117

knowing someone with a history of more than one type of loss (miscarriage, stillbirth, neonatal death and/or other). Additionally, 44 participants (31.9\%) indicated the relationship to the woman they knew with a history of perinatal loss as a family member, $44(31.9 \%)$ as a friend, eight $(5.8 \%)$ as other than a family member or friend and 42 $(30.4 \%)$ as more than one relation (family member, friend and/or other).

\section{Materials}

\section{The Perinatal Grief Scale-Short Form (PGS)}

The PGS is an instrument designed to measure grief following perinatal loss (Toedter, Lasker, \& Janssen, 2001). As a total scale score, the PGS consists of three subscales, namely Active Grief, Difficulty Coping and Despair. The Active Grief subscale presents information on acute, common grief reactions and includes items about sadness, missing the baby and crying for the baby. The latter two subscales measure complicated grief reactions. Difficulty Coping indicates withdrawal and depression and includes items suggesting difficulty in dealing with normal activities and with people. Despair measures feelings of worthlessness and hopelessness. The PGS has a 5-point Likert-type scale varying from strongly agree to strongly disagree. Higher scores reflect more intense grief reactions. This study used a modified version of the 33-item short form. The interest of this study was with others' perceptions of women who have experienced perinatal loss. Thus, the items were modified, with permission from the test developer, from first person narrative to third person narrative (e.g., from "I feel depressed" to "She feels depressed"). The PGS was supported as a psychometrically sound test (Toedter et al., 2001); however, these findings do not apply to the modified version used in this study.

\section{The Perceptions of Medical Patients Questionnaire (PMPQ)}

The PMPQ is a measure used to assess perceptions of medical patients' psychological distress level as well as perceptions of patients' social, emotional and physical adjustment to the medical issue and treatment. The developer of the questionnaire, Hailey (1989) and her research team have used this measure repeatedly to work at achieving a better understanding of others' perceptions of medical patients (e.g. Hailey, 1989; Hardin \& Hailey, 1993).

The PMPQ has 12 questions that are answered using a 5-point Likert-type scale. Questions 6 through 12 have been adapted from the original questionnaire referencing cancer to referencing pregnancy loss. Prior to completing the PMPQ, participants were instructed to imagine they were preparing to visit the woman who experienced perinatal loss. Eight questions ask the participants to rate their expectations of the 
woman (e.g. "How depressed would you expect her to be?") and four questions ask the participants to predict their own feelings in response to the woman (e.g. "How difficult would it be for you to go visit her by yourself?"), (Hailey, Antick, Billie \& Butler, 1994). Scores from Items 3, 4, 6 and 12 that reference the participants' own feelings towards the woman were summed to represent an exploratory subscale measuring discomfort visiting the woman. With the reversal of Items 5 and 7, higher scores indicate more negative perceptions about the woman or higher levels of discomfort, whereas lower scores indicate more positive perceptions about the woman or higher levels of comfort. The PMPQ does not yield a total score; each item is scored and interpreted individually. Currently, no psychometric data is available pertaining to this instrument.

\section{Demographic Self-report Form}

Participants were asked to identify their gender, race/ethnicity and history of perinatal loss (i.e., whether they had experienced perinatal loss or if they had known someone who had experienced perinatal loss) on the demographic self-report form. Additionally, space was provided for participants to write any comments they had about the study or their direct or indirect experiences with perinatal loss.

\section{Procedure \& Research Design}

After running a power analysis, it was determined that 156 participants were needed to detect an effect size of .40 at .80 power. Participants were recruited from the Pacific University, undergraduate and graduate populations via email. Specifically, an email was sent to a contact person from each undergraduate and graduate department requesting the invitation for participation be forwarded to his/her respective student population.

Data were collected in individual and group settings. Prospective participants were verbally informed of the nature of the study and the procedures taken to ensure confidentiality and were then asked to read and sign an informed consent form if they wished to participate. Participants were also asked to provide contact information if they were interested in learning the results of the study. After completion of the informed consent, they were presented with a research packet containing the demographic self-report form, one of two vignettes describing a hypothetical woman who experienced perinatal loss, the PGS and the PMPQ. The vignettes varied in regard to type of perinatal loss, miscarriage or stillbirth.

Participants were instructed to think of the woman as a close family friend while reading the vignette and to imagine how she would 
Plagge \& Antick: PERCEPTIONS OF PERINATAL LOSS 119

be feeling. Participants then completed the PGS based on their perceptions of how the expectant mother may have felt within the week following the loss. Afterwards, participants completed the PMPQ while thinking of their own expectations of and feelings about the woman described in the vignettes.

\section{Data Analysis}

Coded data were entered into SPSS to obtain descriptive statistics and analysis. Analysis included two independent t-tests to determine whether differences existed between the group that reviewed the miscarriage vignette and the group that reviewed the stillbirth vignette. The first independent t-test was conducted to determine whether scores differed between the two groups on the total PGS score. The dependent variable was the total PGS score reflecting perceived grief. The second independent t-test was conducted to determine whether the summed scores from the four items (Items 3, 4, 6 and 12) on the PMPQ differed between the two groups reflecting discomfort visiting the woman in the vignette. These four items appear to measure the expected discomfort visiting the woman in the vignette and, therefore, were summed to represent an exploratory subscale.

\section{RESULTS}

\section{Perceptions of Grief}

An independent t-test was conducted to test whether participant ratings of the stillbirth vignette reflected higher scores of perceived grief than participant ratings of the miscarriage vignette as measured by the total PGS score. Levene's Test for Equality of Variances was not violated $(F=3.32, p=.070)$; therefore, equal variances between groups were assumed. Results $(t(167)=-4.09, p<.001)$ indicated a statistically significant mean difference between the group that reviewed the stillbirth vignette $(M=123.42 ; S D=14.36)$ and the group that reviewed the miscarriage vignette $(M=113.23 ; S D=17.91)$. These findings support the research hypothesis that participant ratings of the stillbirth vignette reflected higher scores of perceived grief than participant ratings of the miscarriage vignette.

\section{Expected Discomfort}

An independent t-test was conducted to test whether participant ratings of the stillbirth vignette reflected higher scores on a scale of expected discomfort visiting the woman than participant ratings of the miscarriage vignette as measured by an exploratory subscale of the PMPQ. Levene's Test for Equality of Variances was not violated $(F=.56$, 
$p=.457)$; therefore, equal variances between groups were assumed. Results $(t(166)=-4.14, p<.001)$ indicated a statistically significant mean difference between the group that reviewed the stillbirth vignette $(M=$ $12.32 ; S D=2.97)$ and the group that reviewed the miscarriage vignette $(M=10.49 ; S D=2.76)$. These findings support the research hypothesis that stillbirth group participants expected higher discomfort than miscarriage group participants.

\section{Perceptions of Fault}

Although a hypothesis was not generated for Item 9 of the PMPQ, which measures perceived fault of the loss attributed to the woman in the vignette, a post-hoc independent t-test was conducted to determine whether a difference between groups existed in participant ratings of those who reviewed the stillbirth vignette and those who reviewed the miscarriage vignette. Levene's Test for Equality of Variances was violated $(F=10.16, p=.002)$; therefore, equal variances between groups were not assumed. Results $(t(129)=-1.62, p=.108)$ indicated no statistically significant mean difference between the group that reviewed the stillbirth vignette $(M=1.19 ; S D=.65)$ and the group that reviewed the miscarriage vignette $(M=1.06 ; S D=.36)$. Thus, no difference in perceived fault was evident between participant ratings of the stillbirth vignette and participant ratings of the miscarriage vignette.

\section{Qualitative Comments}

Many of the participants made written comments throughout the study. The majority of participants who commented wrote about a family member who had experienced perinatal loss. However, others also wrote about a friend or acquaintance they knew who had experienced perinatal loss and one participant made a comment about her own experience of perinatal loss. Comments made by some of the participants are presented below.

My cousin was carrying the fetus for about three months. She took great care of herself and had a miscarriage. The after effect she suffered was an increase in alcohol [use]. Currently, she still has not been able to have a child and adopted her niece.

My mom miscarried prior to my birth. My parents wanted only one child so I would not exist if that hadn't happened!

My mom's first pregnancy was a miscarriage. Although it was extremely difficult for her, my brother would not be here if the first baby [had] lived so she looks at it as 'God's plan.' 
Plagge \& Antick: PERCEPTIONS OF PERINATAL LOSS 121

My cousin's first born was a miscarriage which was a hard issue for my family. We are very close.

My family has a history of miscarriages and stillbirths. All female members of my family have had miscarriages and two have had stillbirths.

This was a family friend and [she] was able to get pregnant and have children at other times.

SIDS [Sudden Infant Death Syndrome] really devastated my friend but she has two children and is very happy. She [did] not mind talking about the death of her son two weeks after he was born and she is at peace with it now.

I had miscarried at 10 weeks about two and half years ago. My best friend miscarried about 2 years ago. We both became pregnant and carried our babies full-term soon after our miscarriages.

\section{DISCUSSION}

The results of this study indicate that there are significant differences in how young adult women perceive the loss of a pregnancy, according to gestational maturity. A stillbirth vignette prompted expectations of more grief than did a vignette of a pregnancy loss when it was classified as a miscarriage. These data also suggest that women who represent the support network for a mother who experiences perinatal loss have significantly greater expectations of discomfort visiting a woman who experienced stillbirth than a woman who experienced miscarriage. These findings are consistent with several studies in the perinatal loss literature regarding women's experience of grief and social support following perinatal loss (e.g., Cuisinier et al., 1993; Van \& Meleis, 2002; Woods \& Woods, 1997).

\section{Perceptions of Grief}

Participant ratings of the stillbirth vignette reflected higher scores of perceived grief than participant ratings of the miscarriage vignette, therefore, suggesting that female friends and family members likely perceive stillbirth as more severe than miscarriage and, consequently, associate a greater degree of grief with stillbirth than miscarriage. This finding is supported by researchers (Cuisinier et al., 1993; Van \& Meleis, 2002) who have suggested that society, including 
healthcare professionals, tend to view stillbirth as a more significant loss than miscarriage. Research has revealed that support varies in relation to time of loss (Cuisinier et al., 1993; Van \& Meleis, 2002) and, thus, type of loss. When individuals do not view perinatal loss as a real life loss and instead view it as, for example, a routine procedure or failed conception, it is likely that they will not have much empathy for the woman who experienced the loss and, in turn provide little support. It is likely that individuals provide varying types and amounts of support based on the meaning they attach to the loss. Hailey and colleagues' (1994) findings further support the possibility that people provide more support to individuals when they attribute more meaning and severity to their experience.

\section{Expected Discomfort}

Participant ratings of the stillbirth vignette reflected higher scores of expected discomfort when visiting the woman than participant ratings of the miscarriage vignette, therefore, suggesting that female friends and family members expect to experience more discomfort visiting a woman who experienced stillbirth than a woman who experienced miscarriage. Perhaps, because participants associate a greater degree of grief associated with stillbirth than miscarriage, they also expect a greater degree of discomfort visiting a woman in more grief.

While the authors of this study cannot conclude that high levels of perceived grief will translate to support offered and high levels of expected discomfort will translate to avoidance, it is hypothesized that participants may be more likely to visit a woman who experienced miscarriage than a woman who experienced stillbirth; and participants may provide more support to those they perceive in more grief than others, despite expecting more discomfort while visiting them. Future research may wish to examine whether the level of grief associated with perinatal loss better predicts the support provided than the level of expected discomfort.

\section{A Supplemental Finding}

A finding of particular interest was revealed through post hoc analysis. In regard to the extent of perceived fault pertaining to the loss, no significant difference was evident. The means for each group were 1.06 (miscarriage vignette) and 1.19 (stillbirth vignette), which both fall between "not at all her fault" and "a little her fault." Therefore, this finding likely suggests that women in the support network attribute relatively low levels of fault with either loss. This finding is contrary to many studies in the medical literature, in which medical patients have 
Plagge \& Antick: PERCEPTIONS OF PERINATAL LOSS 123

often been perceived as at fault for their illness (e.g. Andsager, Hust, \& Powers, 2000; Chapple, Ziebland, \& McPherson, 2004; Richards, Reid, \& Watt, 2003). Richards and colleagues (2003) reported that ill health is often perceived to be closely linked with individual responsibility. Thus, it is likely that knowledge that behaviors and lifestyle choices can affect a woman's health and consequently her unborn child's health, may have influenced participants to attribute some fault to the woman in the vignette. This difference may also be due, in part, to gender differences in that individuals may be more willing to blame women rather than men in certain cases. The large pervasiveness of mother blaming is an example of this (Jackson \& Mannix, 2004) and it is possible that mother blaming played a part in participants' ratings of fault attributed to the women who experienced perinatal loss in the vignettes.

It is also interesting to note that the perinatal loss literature indicates that women who experience perinatal loss often report selfblame (Bennett et al., 2005; De Frain et al., 1990; McGreal, Evans, \& Burrows, 1998; Woods \& Woods, 1997). Self-blame is additionally reported in studies of medical patients, such as cancer and heart disease patients. While low levels of blame were attributed within this study, some fault was still attributed and thus, it appears that fault is attributed to perinatal loss or illness, regardless of whether the person is a patient or witness. Self-blame may also be the result of medical knowledge concerning ill effects of risk behaviors on health and illness.

\section{Qualitative Comments}

The comments participants shared show that several of the participants were aware of family members, friends, or acquaintances they knew who had experienced perinatal loss. Some of the comments indicated that the participants acknowledged the difficulty of the loss for the woman. However, many who commented also attempted to make light of the loss by explaining how they were able to have children at a later time. Interestingly, Côté-Arsenault and Morrison-Beedy (2001) found that acknowledgment of perinatal loss appeared to validate women's concerns and assured them that others were listening. However, friends and family who made light of their concerns or made trite comments such as "at least you can get pregnant" (Côté-Arsenault \& Morrison-Beedy, 2001, p. 243), were perceived as unhelpful and hurtful. Although the participants in this study did not make comments such as this, the comments they made about being able to become pregnant at a later time may be interpreted as taking away from the initial acknowledgement of the loss and perhaps negatively influencing the support they provided. 


\section{Study Limitations \& Directions for the Future}

The most evident limitation within this study is that the sample consisted of only female participants of a relatively restricted age range. It is important to note that men and specifically the expectant fathers and significant others of expectant mothers, comprise a woman's social network and, therefore, their participation in such a study would provide additional knowledge into perceptions of women who have experienced perinatal loss in regard to grief experienced and expected discomfort. Future research should investigate why it is that women suffer or are perceived to suffer more than men; why expectant fathers receive less support than expectant mothers; and whether male partners and/or significant others provide adequate support to their female partners following perinatal loss.

Other factors affecting generalization include a limited representation of minority ethnic groups; the majority of participants were Caucasian, making up 84.6\% of the participant pool. Also, all 169 participants were either undergraduate or graduate students at the same university with a median age of 25 years old and of the participants, $46.6 \%$ were graduate students studying clinical psychology. It should also be acknowledged that the literature reviewed did not include research outside of North America and therefore, it is likely reflective of North American proclivities, which tend to be less patriarchal than other regions of the world. Thus, the relatively similar demographics of the sample and North American biases further limit the generalizability of the results.

Another critique of this study is that 15 participants indicated a history of perinatal loss, whereas 154 participants indicated no history of perinatal loss. These participants with a history of perinatal loss were randomly dispersed between the two groups (miscarriage vignette, stillbirth vignette). It is possible that these women may have significantly different perceptions of the women who experienced perinatal loss described in the vignettes given their personal experience with similar losses. For instance, Bennett and colleagues (2005) reported that individuals with no prior knowledge or experiential history of perinatal loss are likely to have difficulty empathizing with women who have experienced perinatal loss. Either excluding these women from the study or forming additional groups for these women may have yielded different results, although of note is that the majority of the sample $(81.7 \%)$ reported knowing someone with a history of perinatal loss, suggesting at least some familiarity with the experience. Future research might explore the variable of history of perinatal loss on perceptions of perinatal loss. Lastly, the meaning and image people attach to pregnancy at varying stages could also be explored. 
Plagge \& Antick: PERCEPTIONS OF PERINATAL LOSS 125

\section{Practical Implications}

Although women experience similar levels of grief regardless of the type of perinatal loss experienced (Woods \& Woods, 1997), participants associated a more severe grief reaction with women who experienced stillbirth than miscarriage, likely reflecting their own image and meaning of the baby given the gestational age. Because female individuals who compose a mother's social support network likely view miscarriage as less severe than stillbirth, it is likely that they provide less support to women who experience miscarriage. Poor social support is related to higher grief reactions and more severe psychiatric symptoms following perinatal loss (Forrest et al., 1982; Hughes \& Riches, 2003; Janssen et al., 1997). If the level of support individuals provide is dependent upon perceptions of perinatal loss, it is important that accurate knowledge of the grief process following varying types of perinatal loss is dispersed to improve the support provided to these women and possibly prevent prolonged and intensified emotional disturbances.

Although this study has limitations, one important implication is its contribution to the existing literature regarding perceptions individuals actually have of women who experience perinatal loss and how those perceptions compare with actual differences reported by the mothers who have experienced perinatal loss. Once researchers have a better understanding of people's existing perceptions, it becomes feasible to develop interventions and education programs designed to contest misperceptions and promote accurate views of expectant mothers who have experienced perinatal loss. As perinatal loss occurs so frequently, it stands to reason that everyone will eventually find themselves in either the role of experiencing perinatal loss or the support system. It is the authors' hope that this study will encourage further research in this field that will improve support for women who experience perinatal loss as well as for family and friends who are also affected by such losses.

\section{REFERENCES}

Andsager, J. L., Hust, S. J. \& Powers A. (2000). Patient-blaming and representation of risk factors in breast cancer images. Women $\mathcal{E}$ Health, 31, 57-79.

Bennett, S. M., Litz, B. T., Sarnoff Lee, B. \& Maguen, S. (2005). The scope and impact of perinatal loss: Current status and future directions. Professional Psychology: Research \& Practice, 36, 180-187.

Chapple A., Ziebland S. \& McPherson A. (2004). Stigma, shame and blame experienced by patients with lung cancer: Qualitative study. British Medical Journal, 328, 1470-1475. 
Côté-Arsenault, D., Bidlack, B. \& Humm, A. (2001). Women's emotions \& concerns during pregnancy following perinatal loss. American Journal of Maternal Child Nursing, 26, 128-134.

Côté-Arsenault, D. \& Morrison-Beedy, D. (2001). Women's voices reflecting changed expectations for pregnancy after perinatal loss. Journal of Nursing Scholarship, 33, 239-244.

Cuisinier, M. C. J., Kuijpers, J. C., Hoogduin, C. A. L., de Graauw, C. P. H. M. \& Janssen, H. J. E. M. (1993). Miscarriage and stillbirth: Time since the loss, grief intensity \& satisfaction with care. European Journal of Obstetrics \& Gynecology \& Reproductive Biology, 52, 163-168.

De Frain, J., Martens L., Stork, J. \& Stork, W. (1990). The psychological effects of a stillbirth on surviving family members. Omega Journal of Death \& Dying, 22, 81-108.

DiMarco, M. A., Menke, E. M. \& McNamara, T. (2001). Evaluating a support group for perinatal loss. American Journal of Maternal Child Nursing, 26, 135-140.

Forrest, G. C., Standish, E., \& Baum, J. D. (1982). Support after perinatal death: A study of support and counseling after perinatal bereavement. British Medical Journal, 285, 1475-1479.

Hailey, B. J. (1989). A comparison of perceptions of breast cancer patients with those of other female patients. Journal of Psychosocial Oncology, 7, 91-101.

Hailey, B. J., Antick, J. R., Billie, S. \& Butler, M. (1994). How breast cancer patients are perceived: Effects of treatment, method, age and time since surgery. Psychooncology, 3, 321-328.

Hardin, K. N., \& Hailey, B. J. (1993). Health care professionals' perceptions of seriously ill women. Health Care for Women International, 14, 7-16.

Hughes, P. \& Riches, S. (2003). Psychological aspects of perinatal loss. Current Opinion in Obstetrics and Gynecology, 15, 107-111.

Hughes, P., Turton, P., Hopper, E. \& Evans, C. D. (2002). Assessment of guidelines for good practice in psychosocial care of mothers after stillbirth: A cohort study. Lancet, 360, 114-118.

Jackson D. \& Mannix J. (2004). Giving voice to the burden of blame: A feminist study of mothers' experiences of mother blaming. International Journal of Nursing Practice, 10, 150-158.

Janssen, H. J. E. M., Cuisinier, M. C. J., de Graauw, K. P. H. M. \& Hoogduin, K. A. L. (1997). A prospective study of risk factors predicting grief intensity following pregnancy loss. Archives of General Psychiatry, 54, 56-61.

Kavanagh, B. (2002). Women, men and stillbirth: How do they cope? The Practising Midwife, 5, 18-20. 
Kochanek, K. D., Murphy, S. L. anderson, R. N. \& Scott, C. (2004). Deaths: Final data for 2002. In National Vital Statistics Reports, 53 (5). Hyattsville, MD: National Center for Health Statistics.

Madden, M. E. (1994). The variety of emotional reactions to miscarriage. Women \& Health, 21, 85-104.

McGreal, D., Evans, B. J. \& Burrows, G. D. (1998). Gender differences in coping following loss of a child through miscarriage or stillbirth: A pilot study. Stress Medicine, 13, 159-165.

Rajan, L. (1994). Social isolation and support in pregnancy loss. Health Visitor, 67, 97-101.

Richards H., Reid M. \& Watt, G. (2003). Victim-blaming revisited: A qualitative study of beliefs about illness causation and responses to chest pain. Family Practice, 20, 711-716.

Swanson, K. M. (2000). Predicting depressive symptoms after miscarriage: a path analysis based on the Lazarus paradigm. Journal of Women's Health \& Gender-Based Medicine, 9, 191-206.

Toedter, L. J., Lasker, J. N. \& Janssen, H. J. (2001). International comparison of studies using the perinatal grief scale: A decade of research on pregnancy loss. Death Studies, 25, 205-228.

Turton, P., Hughes, P., Evans, C. D. H. \& Fainman, D. (2001). Incidence, correlates \& predictors of post-traumatic stress disorder in the pregnancy after stillbirth. British Journal of Psychiatry, 178, 556-560.

Van, P. \& Meleis, A. I. (2002). Coping with grief after involuntary pregnancy loss: Perspectives of African American women. Journal of Obstetric, Gynecologic \& Neonatal Nursing, 32, 28-39.

Wallerstedt, C., Lilley, M. \& Baldwin, K. (2003). Interconceptional counseling after perinatal \& infant loss. Journal of Obstetric, Gynecologic \& Neonatal Nursing, 32, (533-542).

Woods, J. R. \& Woods J. L. E. (Eds.). (1997). Loss During Pregnancy or in the Newborn Period. Pitman, NJ: Jannetti Publications, Inc. 